\title{
Is heat pain detection threshold associated with the area of secondary hyperalgesia following brief thermal sensitization? A study of healthy volunteers - design and detailed plan of analysis
}

Morten Sejer Hansen ${ }^{1 *}$, Jørn Wetterslev ${ }^{2}$, Christian Bressen Pipper ${ }^{3}$, Mohammad Sohail Asghar $^{1}$ and Jørgen Berg Dahl ${ }^{4}$

\begin{abstract}
Background: Several factors are believed to influence the development and experience of pain. Human clinical pain models are central tools, in the investigation of basic physiologic pain responses, and can be applied in patients as well as in healthy volunteers. Each clinical pain model investigates different aspects of the human pain response.

Brief thermal sensitization induces a mild burn injury, resulting in development of primary hyperalgesia at the site of stimulation, and secondary hyperalgesia surrounding the site of stimulation. Central sensitization is believed to play an important role in the development of secondary hyperalgesia; however, a possible association of secondary hyperalgesia following brief thermal sensitization and other heat pain models remains unknown. Our aim with this study is to investigate how close the heat pain detection threshold is associated with the size of the area of secondary hyperalgesia induced by the clinical heat pain model: Brief thermal sensitization.
\end{abstract}

Methods and design: We aim to include 120 healthy participants. The participants will be tested on two separate study days with the following procedures: i) Brief thermal sensitization, ii) heat pain detection threshold and iii) pain during thermal stimulation. Additionally, the participants will be tested with the Pain Catastrophizing Scale and Hospital Anxiety and Depression Scale questionnaires.

We conducted statistical simulations based on data from our previous study, to estimate an empirical power of 99. $9 \%$ with a of 0.05 . We define that an $R^{2}<0.25$ and predictive intervals larger than $+/-150 \mathrm{~cm}^{2}$ are indications of a weak association.

Discussion: The area of secondary hyperalgesia may serve as a quantitative measure of the central sensitization induced by cutaneous heat stimulation, and thus may be a biomarker of an individual's pain sensitivity. The number of studies investigating secondary hyperalgesia is growing; however basic knowledge of the physiologic aspects of secondary hyperalgesia in humans is still incomplete. We therefore find it interesting to investigate if HPDT, a known quantitative sensory test, is associated with areas of secondary hyperalgesia following brief thermal sensitization

(Continued on next page)

\footnotetext{
* Correspondence: morten.sejer.hansen@regionh.dk

${ }^{1}$ Department of Anaesthesiology, 4231, Centre of Head and Orthopaedics,

Rigshospitalet, Blegdamsvej 9, Copenhagen 2100, Denmark

Full list of author information is available at the end of the article
} 
(Continued from previous page)

Trial registration: Clinicaltrials.gov (Identifier: NCT02527395). Danish Research Ethics Committee (Identifier: H-8-2014-012). Danish Data Protection Agency (Identifier: 30-1436).

Keywords: Pain, Anaesthesiology, Physiology, Hyperalgesia, Central sensitization, Quantitative sensory testing

\section{Background}

Psychology, physiology, ethnicity, sex and psychosocial background are just some of the factors believed to contribute to the pain experience in the individual. However, the knowledge of the individual mechanisms responsible for the development of acute and chronic pain is still incomplete [1].

Human clinical pain models are central tools, in the investigation of basic physiologic pain responses, and can be applied in patients as well as in healthy volunteers $[2,3]$.

With the pain model brief thermal sensitization (BTS), the skin is heated to $45^{\circ} \mathrm{C}$ for $3 \mathrm{~min}$. resulting in the development of primary hyperalgesia at the site of stimulation, and secondary hyperalgesia surrounding the site of stimulation [4-8]. The area of secondary hyperalgesia is characterised by reduced thresholds for mechanical stimulation, and the size of the area can be quantified by monofilament stimulation. Current evidence indicates that the development of secondary hyperalgesia to punctate mechanical stimuli following a standardised injury is caused by central changes in response to a conditioning stimulus and transmitted by A-delta fibers [1, 9-11]. The secondary hyperalgesia elicited by a clinical pain model may thus be a result of central sensitization. Central sensitization reflects a functional change in the neuron properties by increased synaptic efficacy and membrane excitability as well as decreased synaptic inhibition [1,9]. This functional change in the neurons and nociceptive pathways results in pain threshold reduction, increased response to noxious stimulation, and prolonged duration of pain following noxious stimulation [1, 9]. Central sensitization encompasses both an early and late phase, with the early phase independent of transcription and the late long-lasting phase transcription dependent [9]. The transcription dependent long lasting phase of central sensitization is believed to play an important role in several different pain conditions and syndromes, e.g. osteoarthritis and fibromyalgia $[1,9,12,13]$.

Secondary hyperalgesia following clinical pain models has been demonstrated to be a robust phenomenon, and can be applied when investigating basic pain physiology in humans $[4-8,14-30]$.

Recently, a meta-analysis [24] of 10 studies (9 published) $[4-6,8,19,21,23,26,30]$ indicated that healthy volunteers had; (I), a large inter-individual variation with regard to the magnitude of the area of secondary hyperalgesia after exposure to identical pain stimuli. (II), that areas of secondary hyperalgesia may be an identifiable phenotypic indicator and that (III), they may be predictive of individual pain responses. Furthermore, in a recently published study [31], results have indicated that increasing heat pain detection threshold (HPDT) may be associated with decreasing size of the area of secondary hyperalgesia. However, the study indicates that HPDT only offers a very modest explanation of the variation in the area of secondary hyperalgesia following standardised burn injury, suggesting that HPDT and secondary hyperalgesia may be two different pain entities.

Moreover, one recent study found indications of structural cerebral differences and differences in neuronal activation to noxious stimulation, in healthy volunteers with a large vs. small area of secondary hyperalgesia following a first degree burn injury [25]. Finally, the study also found suggestions of an association between large areas of secondary hyperalgesia and high anxiety scores.

The different physiologic properties of BTS and HPDT [1, 9-11, 32] suggests that they may be two different pain entities; however the current evidence on the basic physiologic properties of the areas of secondary hyperalgesia in humans remains incomplete. The predictive value of HPDT on postoperative pain [33-35], suggests that HPDT may partly provide insight on an individual's pain sensitivity; however, our previous results indicate that HPDT and the development of secondary hyperalgesia may only be weakly associated. This result challenges the current assumption that areas of secondary hyperalgesia may be a marker for an individual's central sensitization and ultimately the pain sensitivity. This rather surprising result needs to be explored further. A thorough evaluation of the basic physiologic properties in the development of secondary hyperalgesia following BTS is necessary in order to exploit the full potential of this clinical pain model, and may provide novel insight in the development of acute and chronic pain.

In this prospective cohort study we therefore aim to investigate how close the HPDT, evaluated on two separate study days, is associated with the size of the area of secondary hyperalgesia induced by BTS. We hypothesise that HPDT and area of secondary hyperalgesia are two predominantly independent entities, and thus, are weakly associated. 


\section{Methods/design}

The method and design of this study is based upon a previous study by our own research group [31]. The recruiting of study participants and commencement of the study was initiated on October 1, 2015.

\section{Study participants}

Only healthy male volunteers will be included in the study. The investigator will provide the study participants with written and oral information about the study, as well as information concerning possible risk and side effects. The study participants will sign a written informed consent, and will receive EUR 20 (USD 27) per hour for their participation in the study. Inclusion and exclusion criteria are presented in Table 1.

\section{Setting}

The study is conducted in a quiet secluded room (temperature: $22-25{ }^{\circ} \mathrm{C}$ ), where only the study participant and the investigator will be present. The study participants will be in a supine position, lying on their back, during the assessments. The study will be conducted at the Department of Anaesthesiology, 4231, Rigshospitalet, Copenhagen, Denmark.

\section{Study design}

The study is a confirmatory study designed to investigate the association between area of secondary hyperalgesia and HPDT. The study consists of a screening/information day and two study days. To prevent carry-over effects of the applied tests, the screening day and each of the two study days must be separated with a minimum of seven days. The aim with our study is to investigate the association between HPDT and area of secondary hyperalgesia, thus the order of the stimulations (HPDT and BTS) is randomised for each participant for each study day. Pain during $1 \mathrm{~min}$. thermal stimulation (p-TS) is conducted subsequent to BTS and HPDT. Likewise, in order to adjust for possible carry-over effects between HPDT and BTS we conduct experimental pain testing on two separate study days with the experimental pain testing sequence randomised for each study day (see Randomisation, allocation concealment and blinding).

The study participants will thus be tested with three types of clinical pain models on two separate study days. The following clinical pain models will be applied: Brief thermal sensitization (BTS), heat pain detection threshold (HPDT) and pain during thermal stimulation (p-TS) (see Assessments and tests). Furthermore, the study participants will complete the two psychological tests, Pain Catastrophizing Scale (PCS) and Hospital Anxiety and Depression Scale (HADS) (see Assessments and tests).

The screening day and the two identical study days will proceed as follows:

Screening day:

- Oral information regarding the study is delivered by the primary investigator. Informed consent and written authority will be handed out for the participant to sign. The participant will be given time to consider the information.

- Short medical history by the primary investigator. Thorough screening of in- and exclusion criteria. Measurement of height, weight, blood pressure and pulse.

- In order to familiarise the study participant with the types of pain conditioning, the 3 types of pain conditioning test are presented and performed on the study participant.

Table 1 Inclusion and exclusion criteria

\begin{tabular}{|c|c|}
\hline Inclusion criteria & Exclusion criteria \\
\hline Age $\geq 18$ years and $\leq 35$ years & Study participants who cannot cooperate to the test. \\
\hline $\begin{array}{l}\text { Speak and understand the Danish } \\
\text { language }\end{array}$ & $\begin{array}{l}\text { Study participants who have a weekly intake of }>21 \text { units of alcohol, or a have consumed }>3 \text { units of alcohol } \\
\text { within } 24 \text { h before study day. }\end{array}$ \\
\hline Healthy male & Study participants with a substance abuse, assessed by the investigator. \\
\hline \multirow[t]{10}{*}{ Signed informed consent } & Study participants, who have consumed analgesics less than 3 days before study day. \\
\hline & Study participants, who have consumed antihistamines less than $48 \mathrm{~h}$ before study day. \\
\hline & Study participants who have consumed antidepressant medication during the last 30 days before the study \\
\hline & Study participants who have consumed prescription medicine during the last 30 days before the study. \\
\hline & Study participants with neurological illnesses. \\
\hline & Study participants with chronic pain \\
\hline & Study participants with psychiatric diagnoses \\
\hline & Study participants with tattoos on the extremities \\
\hline & Study participants with eczema, wounds or sunburns on the sites of stimulation. \\
\hline & Study participants with a Body Mass Index of $>30 \mathrm{~kg} / \mathrm{m}^{2}$ or $<18 \mathrm{~kg} / \mathrm{m}^{2}$ \\
\hline
\end{tabular}


- Distribution of PCS and HADS (in Danish), together with an opaque envelope, in which the answered tests are to be put, and a sealed envelope will be returned to the primary investigator on the first study day.

- Potentially clarifying questions concerning the study or PCS and HADS are answered.

Study day 1 and 2:

- Inspection of signed informed consent and written authority.

- Study day 1: Hand in of PCS and HADS in an opaque sealed envelope. The envelope is first opened when all study participants have completed study day 1 and 2 .

- The study participant is placed on a bed in the supine position.

- The timeline of tests performed will be as follows:

o Commencement of study 0 min: BTS or HPDT (depending on randomisation)

- At 7 min: BTS or HPDT (depending on randomisation)

$\circ$ At 10 min: $\mathrm{p}$-TS

\section{Assessment and tests}

\section{Brief thermal sensitization (BTS)}

A computer-controlled thermode (Somedic MSA Thermotester $^{\mathrm{mw}}$; size $2.5 \times 5 \mathrm{~cm}$ ) is placed on the participant's skin. The initial temperature of the thermode is $32{ }^{\circ} \mathrm{C}$, and temperature is increased $1{ }^{\circ} \mathrm{C} / \mathrm{s}$ until it reaches $45^{\circ}$ C. The temperature of the thermode remains $45{ }^{\circ} \mathrm{C}$ for $3 \mathrm{~min}$., and while the thermode still has contact with the skin, and has a temperature of $45{ }^{\circ} \mathrm{C}$, the assessment of secondary hyperalgesia is conducted. The assessment of secondary hyperalgesia will approximately take 1-2 min, with a maximum duration of heat stimulation of $5 \mathrm{~min}$. The test is conducted centrally on the anterior part of the right thigh.

\section{Assessment of secondary hyperalgesia}

The area of secondary hyperalgesia is quantified after stimulation with a 19G monofilament (von Frey hair) in 4 linear paths arranged in $90^{\circ}$ around the centre of stimulation. Stimulation will begin $15 \mathrm{~cm}$. from the centre of stimulation and advance in steps of $5 \mathrm{~mm}$. with $1 \mathrm{~s}$ intervals towards the centre of stimulation. When the participant states a clear change in sensation (intense pricking, burning, tenderness) the place will be marked with a felt pen and the transverse and longitudinal axes will be measured with a pliable measuring tape for later rectangular area calculation.

\section{Heat pain detection threshold (HPDT)}

Heat pain detection threshold represents the lowest temperature that is perceived as painful, when heating the skin with the computer-controlled thermode. The initial temperature is $32{ }^{\circ} \mathrm{C}$, and temperature is increased $1{ }^{\circ} \mathrm{C} / \mathrm{s}$. The participant is asked to press a button when the heat is perceived as painful. If $52{ }^{\circ} \mathrm{C}$ (the maximum temperature for the thermode) is reached before the participant's threshold has been registered, the thermode will automatically return to the initial temperature of $32{ }^{\circ} \mathrm{C}$ to prevent excessive tissue damage. The HPDT is calculated as an average of four stimulations. Each stimulation will be performed with an interval of 6-10 s. The test is conducted centrally on the anterior part of the dominant lower arm.

\section{Pain during thermal stimulation ( $p-T S)$}

The computer-controlled thermode is placed on the participant's skin. The initial temperature of the thermode is $32{ }^{\circ} \mathrm{C}$, and temperature is increased $1{ }^{\circ} \mathrm{C} / \mathrm{s}$ until it reaches $45^{\circ} \mathrm{C}$, where it remains for $1 \mathrm{~min}$. During this the participant evaluates the pain with an electronic VAS-scale. The participant will continuously evaluate the pain using the electronic VAS-scale because of the fluctuations in pain intensity during this type of stimulation. The equipment (Somedic USB-VAS) automatically calculates a VAS-score under the curve (VAS-AUC) and a maximum VAS-score for the time period. The participant will not be able to see the computer screen during the measurement, and each pain evaluation will be independent of the previous evaluation. The test is conducted centrally on the anterior part of the nondominant lower arm.

\section{Pain assessment}

Visual analogue scale (VAS), index from 0-100 mm, where $0 \mathrm{~mm}$ represents "no pain", and $100 \mathrm{~mm}$ represents "the worst pain imaginable".

\section{Hospital Anxiety and Depression Scale (HADS)}

HADS is a questionnaire consisting of 14 questions, and is a four-point Likert scale with values from 0-3. HADS can be subdivided into HADS-A, evaluating anxiety, and HADS-D, evaluating depression. The highest achievable score is 42, and a total HADS-score will estimate the participant's level of distress. To evaluate anxiety and depression separately, HADS-A and HADS-D must be evaluated separately, with a maximum score of 21 in the two subtests.

The interpretation of the score in HADS-A and HADS-D is as follows:

- 0-7: Normal

- 8: Mild level of anxiety/depression 
- 11-15: Moderate level of anxiety/depression

- $\geq 16$ : Severe level of anxiety/depression

The HADS questionnaire is to be completed before study day 1.

\section{Pain Catastrophizing Scale (PCS)}

PCS is a questionnaire consisting of 13 questions. PCS is a five-point Likert scale with values from $0-4$, and can be subdivided into 3 subtests, that each evaluates the central elements in catastrophizing: Rumination, magnification and helplessness. The highest achievable score is 52, and with separate evaluation of the three subtests, the three different elements can be assessed individually. To evaluate the three elements separately the 13 questions must be evaluated in the three following subgroups:

- Rumination: The sum of question 8, 9, 10, 11. Maximal sum $=16$

- Magnification: The sum of question 6, 7, 13. Maximal sum $=12$

- Helplessness: The sum of question 1, 2, 3, 4, 5, 12. Maximal sum $=24$

The PCS questionnaire is to be completed before study day 1 .

\section{Randomisation, allocation concealment and blinding}

The three types of clinical pain models consist of BTS, HPDT, and p-TS (see Assessments and tests). p-TS are conducted subsequent to BTS and HPDT. The order of BTS and HPDT is randomised, by a random allocation sequence, computer-generated by Copenhagen Trial Unit and stored in sealed and opaque envelopes to secure adequate allocation concealment.

Completed psychological tests will be kept in opaque sealed envelopes. The blinding will be broken when all volunteers have completed the two study days.

\section{Outcomes}

\section{Primary outcome}

How close is the heat pain detection threshold associated with the size of the area of secondary hyperalgesia induced by BTS? The association will be expressed in $\mathrm{R}^{2}$ and with prediction intervals for the area of BTS given fixed values of HPDT.

\section{Secondary outcomes}

To investigate:
1. How close the VAS-AUC following p-TS is associated with the size of the area of secondary hyperalgesia induced by BTS.

2. How close the Max VAS-score following p-TS is associated with the size of the area of secondary hyperalgesia induced by BTS.

3. How close the score of PCS is associated with the size of the area of secondary hyperalgesia induced by BTS.

4. How close the score of HADS is associated with the size of the area of secondary hyperalgesia induced by BTS.

5. How close the subscales in the two psychological tests (PCS-rumination, PCS-magnification, PCShelplessness, and HADS-Anxiety, HADS-Depression) are associated with the size of the area of secondary hyperalgesia.

\section{Hypothesis}

Our hypothesis is that HPDT and area of secondary hyperalgesia elicited by BTS are two predominantly independent entities, and that the area of secondary hyperalgesia is poorly explained by HPDT. We will take $\mathrm{R}^{2}<0.25$ and predictive intervals larger than $+/-150 \mathrm{~cm}^{2}$ as indications of a weak association.

\section{Simulation based assessment of sample size}

Simulation based power calculations are based on data from our own previous study [31]. In particular power calculations are based on a linear relationship between BTS and HPDT derived from inference of data from this previous study.

With $\alpha$ of 0.05 and 120 participants we estimated an empirical power of over $99.9 \%$ using simulations. This provides adequate power to confirm if heat pain detection threshold and area of secondary hyperalgesia following BTS are to large extent independent entities, and thus weakly associated.

Please see the online available Additional file 1 (intrep3-ver3-2015) for the full simulation study.

\section{Data analysis plan}

\section{Plan of statistical analysis of primary outcome}

The association of HPDT with the area of secondary hyperalgesia will be investigated by linear regression on the estimated best linear unbiased predictors (EBLUPS) of the individual area of secondary hyperalgesia. The individual area of secondary hyperalgesia will be adjusted with the body surface area of the individual participant. Significance of the predictor will be assessed by ANOVA methods. The ability of the HPDT to predict individual variations in areas of secondary hyperalgesia will be investigated by linear regression on the EBLUPS of the individual area of secondary hyperalgesia. Significance of 
the predictor will be assessed by ANOVA methods and the predictive ability will be quantified by summary of prediction error including $95 \%$ prediction interval.

Relevant examples will be presented illustrating how accurate HPDT predicts secondary hyperalgesia in individual patients or vice versa.

Subgroup analyses based will be performed if at least $15 \%$ of the study population differs in ethnicity.

\section{Plan of statistical analysis of secondary outcomes}

For each of the 5 secondary outcomes the association of VAS-AUC (following p-TS), Max VAS-score (following p-TS), PCS, HADS, and subscales of PCS and HADS with the area of secondary hyperalgesia will be investigated by linear regression on the estimated best linear unbiased predictors (EBLUPS) of the individual area of secondary hyperalgesia. Significance of the predictor will be assessed by ANOVA methods. In case of significance, the ability of the five variables to predict individual variations in areas of secondary hyperalgesia will be investigated by linear regression on the EBLUPS of the individual area of secondary hyperalgesia. Significance of the predictors will be assessed by ANOVA methods and their predictive abilities will be quantified by various summaries of prediction errors including $95 \%$ prediction intervals.

Relevant examples will be presented illustrating how accurate the five variables predict secondary hyperalgesia in individual participants or vice versa.

\section{Missing data}

For all analyses, an intention-to-test (ITT) analysis will be performed including all subjects that participated in the first study day. Analyses will be based on all observed data. If missingness exceeds $5 \%$ and there is indication of violation of "missing completely at random" (MCAR) by a statistical significant Littles test, a sensitivity analysis based on an appropriate model for missingness at random (MAR) or not at random (MNAR) will be performed.

\section{Statistical significance}

All $p$-values are evaluated at a $5 \%$ significance level. $P$ values for multiple comparisons are adjusted for multiple testing by means of single-step correction [36].

\section{Software}

All analyses will be made using the open source statistical programming environment $\mathrm{R}$ [37].

\section{Ethics}

The applied methods do not cause damage to the skin or have any other long-term adverse effects. In rare cases, changes comparable with a first degree sun burn may appear.

The study will be conducted in accordance with the principles of the Declaration of Helsinki. Written informed consent will be obtained from all the study participants. The participants will be aware of the rare possibility of a first degree burn prior to consenting.

The protocol is approved by the local Danish Research Ethics Committees (Identifier: H-8-2014-012) and the Danish Data Protection Agency (Identifier: 30-1436). The study is also reported on the international database clinicaltrials.gov (Identifier: NCT02527395).

\section{Discussion}

In the present study we aim to investigate how close the HPDT is associated with the size of the area of secondary hyperalgesia induced by BTS. Based on a previous study [31], we hypothesise that HPDT and area of secondary hyperalgesia elicited by BTS are two predominantly independent entities, and that the area of secondary hyperalgesia is poorly explained by HPDT. We estimate that an $R^{2}<0.25$ and predictive intervals larger than $+/-150 \mathrm{~cm}^{2}$ are indications of a weak association. Because we hypothesise that HPDT and the area of secondary hyperalgesia following BTS are possibly two different pain entities we need a high statistical power to ensure that our result is valid. We conducted statistical simulations with the raw data from our previous study [31], and have estimated that with 120 participants we achieve an empirical power of $99.9 \%$. With an $\alpha$ of 0.05 and an estimated empirical power of almost $100 \%$ we feel confident that the risk of type 2 error is minimal.

The area of secondary hyperalgesia may serve as a quantitative measure of the central sensitization induced by cutaneous heat stimulation, and thus may be a biomarker of an individual's pain sensitivity. The stimuli following HPDT and BTS are transmitted in A-delta and C-fibres [32].

With $1{ }^{\circ} \mathrm{C} / \mathrm{s}$. increase in temperature HPDT primarily activates $\mathrm{C}$ polymodale nociceptive afferents, and to a lesser degree A-delta fibres [32]. However, the development of secondary hyperalgesia to punctate mechanical stimuli is centrally mediated and occurs as a result of central plasticity, and has been demonstrated to involve nerve signals primarily in A-fiber nociceptors, not C-fibres [1, 9-11]. HPDT has been demonstrated to be highly reproducible, and may serve as a predictor of pain responses following surgery [33-35], but the current scientific evidence investigating the predictive value of areas of secondary hyperalgesia remains sparse. Thus, if this study demonstrates a weak association between HPDT and secondary hyperalgesia, it does not mean that secondary hyperalgesia as a biomarker for pain sensitivity is useless, just that HPDT and secondary hyperalgesia following BTS may be two different pain 
entities, and express different aspects of pain physiology. Studies investigating the value of secondary hyperalgesia in predicting postoperative pain are warranted in order to clarify this aspect.

Ethnicity, sex, obesity and hormone cycle are all factors that may influence pain sensitivity [20,38-50]. In this study we aim to investigate the association between HPDT and secondary hyperalgesia following BTS, and in order to isolate a possible association between HPDT and secondary hyperalgesia we chose to include healthy male volunteers only. The inclusion of a homogenous male population enables us to focus on the association between HPDT and secondary hyperalgesia, instead of the influence of the individual participant characteristics. This may also be a limitation since the result of the study is primarily applicable in male volunteers. However, to our knowledge, this is the first study investigating the association between HPDT and the area of secondary hyperalgesia following a cutaneous heat injury, and, thus we need to limit known and unknown variables that may influence the primary outcome and distort the signal.

Several clinical studies have demonstrated associations between pain and psychological variables [51-54]; however, the influence of psychological variables on experimental pain still remains unclear [55]. In this study we also aim to investigate a possible association between secondary hyperalgesia and anxiety, depression and pain catastrophizing. One study by Salomon et al. [56] demonstrated that a cognitive-behavioral intervention reducing PCS may reduce the size of the area secondary hyperalgesia. Salomon et al. suggests that central sensitization can be modified by intervening and altering the pain-relating thoughts, and, thus suggests a possible association between the size of secondary hyperalgesia areas and PCS.

The number of studies investigating secondary hyperalgesia as a biomarker for central sensitization and pain sensitivity is growing; however basic knowledge on the physiologic aspects of secondary hyperalgesia in humans is still incomplete. We therefore find it interesting to investigate if HPDT, a known quantitative sensory test [57], is associated with areas of secondary hyperalgesia following BTS.

\section{Additional file}

Additional file 1: Simulation study. (PDF 204 kb)

\footnotetext{
Abbreviations

${ }^{\circ} \mathrm{C}$, Degrees celsius; ANOVA, Analysis of variance; AUC, Area under the curve; BTS, Brief thermal sensitization; Cm, Centimetres; EBLUPS, Estimated best linear unbiased predictors; EUR, Euro; HADS, Hospital anxiety and depression scale; HPDT, Heat pain detection threshold; MAR, Missing at random; MCAR, Missing completely at random; Min, minutes; mm, Millimetre; MNAR, Missing not at random; PCS, Pain catastrophizing scale; $\mathrm{p}-\mathrm{TS}$, Pain during $1 \mathrm{~min}$. thermal stimulation; Sec, Seconds; USD, United States Dollars; VAS, Visual analog scale.
}

Acknowledgments

Not applicable.

\section{Funding}

This work is supported by grants from the Augustinus foundation, Toyota Fonden - Denmark, and the Aase and Ejnar Danielsen's foundation. The funders had no role in the conception or design of the study, or on the decision to publish the results.

\section{Availability of data and materials}

All relevant data will be made available upon publication of the study results.

\section{Authors' contributions}

MSH contributed to the conception and design of the study, manuscript writing and final approval of the manuscript. JBD, MSA and JW contributed to the conception and the design of the study, critical revision of the manuscript, and final approval of the manuscript. CBP contributed to the design of the study, with emphasis on the statistical analysis and sample size analyses, critical revision of the manuscript and final approval of the study. All authors have read and approved the final manuscript.

\section{Competing interests}

The authors declare that they have no competing interests.

\section{Consent for publication}

Not applicable.

\section{Ethics approval and consent to participate}

The protocol is approved by the local Danish Research Ethics Committees (Identifier: H-8-2014-012) and the Danish Data Protection Agency (Identifier: 30-1436).

The study participants will sign a written informed consent, and will receive EUR 20 (USD 27) per hour for their participation in the study.

\section{Author details}

${ }^{1}$ Department of Anaesthesiology, 4231, Centre of Head and Orthopaedics, Rigshospitalet, Blegdamsvej 9, Copenhagen 2100, Denmark. ${ }^{2}$ Copenhagen Trial Unit, Centre for Clinical Intervention Research, dep. 7812, Blegdamsvej 9, Copenhagen 2100, Denmark. ${ }^{3}$ Section of Biostatistics, Faculty of Health, Copenhagen University, Øster Farigmagsgade 5, Copenhagen 1014, Denmark. ${ }^{4}$ Department of Anaesthesiology, dep. Z, Bispebjerg Hospital, Bispebjerg Bakke 23, Copenhagen 2400, Denmark.

Received: 10 October 2015 Accepted: 15 May 2016

Published online: 31 May 2016

\section{References}

1. Woolf CJ. Central sensitization: implications for the diagnosis and treatment of pain. Pain. 2011;152(3 Suppl):S2-15. doi:10.1016/j.pain.2010.1009.1030. Epub 2010 Oct 1018.

2. Handwerker HO, Kobal G. Psychophysiology of experimentally induced pain. Physiol Rev. 1993;73(3):639-71.

3. Reddy KS, Naidu MU, Rani PU, Rao TR. Human experimental pain models: a review of standardized methods in drug development. J Res Med Sci. 2012; 17(6):587-95.

4. Dirks J, Petersen KL, Dahl JB. The heat/capsaicin sensitization model: a methodologic study. J Pain. 2003;4(3):122-8.

5. Dirks J, Petersen KL, Rowbotham MC, Dahl JB. Gabapentin suppresses cutaneous hyperalgesia following heat-capsaicin sensitization. Anesthesiology. 2002;97(1):102-7.

6. Frymoyer AR, Rowbotham MC, Petersen KL. Placebo-controlled comparison of a morphine/dextromethorphan combination with morphine on experimental pain and hyperalgesia in healthy volunteers. J Pain. 2007;8(1):19-25

7. Petersen KL, lyengar S, Chappell AS, Lobo ED, Reda H, Prucka WR, et al. Safety, tolerability, pharmacokinetics, and effects on human experimental pain of the selective ionotropic glutamate receptor 5 (iGluR5) antagonist LY545694 in healthy volunteers. Pain. 2014;155(5):929-36.

8. Petersen $\mathrm{KL}$, Meadoff T, Press S, Peters MM, LeComte MD, Rowbotham MC. Changes in morphine analgesia and side effects during daily subcutaneous administration in healthy volunteers. Pain. 2008;137(2):395-404. 
9. Latremoliere A, Woolf CJ. Central sensitization: a generator of pain hypersensitivity by central neural plasticity. J Pain. 2009;10(9):895-926. doi:10.1016/j.jpain.2009.1006.1012.

10. Ziegler EA, Magerl W, Meyer RA, Treede RD. Secondary hyperalgesia to punctate mechanical stimuli. Central sensitization to A-fibre nociceptor input. Brain. 1999;122(Pt 12):2245-57.

11. Treede RD. Chapter 1 Pain and hyperalgesia: definitions and theories. Handb Clin Neurol. 2006;81:3-10.

12. Arendt-Nielsen L, Yarnitsky D. Experimental and clinical applications of quantitative sensory testing applied to skin, muscles and viscera. J Pain. 2009;10(6):556-72.

13. Edwards RR. Individual differences in endogenous pain modulation as a risk factor for chronic pain. Neurology. 2005;65(3):437-43.

14. Mathiesen $\mathrm{O}$, Imbimbo BP, Hilsted KL, Fabbri L, Dahl JB. CHF3381, a Nmethyl-D-aspartate receptor antagonist and monoamine oxidase-A inhibitor, attenuates secondary hyperalgesia in a human pain model. J Pain. 2006:7(8):565-74.

15. Naert AL, Kehlet $H$, Kupers R. Characterization of a novel model of tonic heat pain stimulation in healthy volunteers. Pain. 2008;138(1):163-71. doi:10. 1016/j.pain.2007.1011.1018. Epub 2008 Jan 1022.

16. Petersen $\mathrm{KL}$, Rowbotham MC. A new human experimental pain model: the heat/capsaicin sensitization model. Neuroreport. 1999;10(7):1511-6.

17. Staahl C, Olesen AE, Andresen T, Arendt-Nielsen L, Drewes AM. Assessing efficacy of non-opioid analgesics in experimental pain models in healthy volunteers: an updated review. Br J Clin Pharmacol. 2009. 68(3):322-41. doi:10.1111/j.1365-2125.2009.03433.x.

18. Cavallone LF, Frey K, Montana MC, Joyal J, Regina KJ, Petersen KL, et al. Reproducibility of the heat/capsaicin skin sensitization model in healthy volunteers. J Pain Res. 2013;6:771-84.

19. Dirks J, Petersen KL, Rowbotham MC, Dahl JB. Effect of systemic adenosine on pain and secondary hyperalgesia associated with the heat/capsaicin sensitization model in healthy volunteers. Reg Anesth Pain Med. 2001;26(5):414-9.

20. Jensen MT, Petersen KL. Gender differences in pain and secondary hyperalgesia after heat/capsaicin sensitization in healthy volunteers. J Pain. 2006;7(3):211-7.

21. Mikkelsen S, Dirks J, Fabricius P, Petersen KL, Rowbotham MC, Dahl JB. Effect of intravenous magnesium on pain and secondary hyperalgesia associated with the heat/capsaicin sensitization model in healthy volunteers. $\mathrm{Br}$ J Anaesth. 2001;86(6):871-3.

22. Petersen $\mathrm{KL}$, Brennum J, Dahl JB. Experimental evaluation of the analgesic effect of ibuprofen on primary and secondary hyperalgesia. Pain. 1997;70(2-3):167-74.

23. Petersen $\mathrm{KL}$, Jones $B$, Segredo $V$, Dahl JB, Rowbotham MC. Effect of remifentanil on pain and secondary hyperalgesia associated with the heat-capsaicin sensitization model in healthy volunteers. Anesthesiology. 2001;94(1):15-20.

24. Werner MU, Petersen KL, Rowbotham MC, Dahl JB. Healthy volunteers can be phenotyped using cutaneous sensitization pain models. PLoS One. 2013;8(5):e62733. doi:10.61371/journal.pone.0062733. Print 0062013.

25. Asghar MS, Pereira MP, Werner MU, Martensson J, Larsson HB, Dahl JB. Secondary hyperalgesia phenotypes exhibit differences in brain activation during noxious stimulation. PLoS One. 2015;10(1):e0114840.

26. Dirks J, Fabricius $\mathrm{P}$, Petersen KL, Rowbotham MC, Dahl JB. The effect of systemic lidocaine on pain and secondary hyperalgesia associated with the heat/capsaicin sensitization model in healthy volunteers. Anesth Analg. 2000;91(4):967-72.

27. Moiniche S, Dahl JB, Kehlet $\mathrm{H}$. Time course of primary and secondary hyperalgesia after heat injury to the skin. Br J Anaesth. 1993;71(2):201-5.

28. Pedersen $\mathrm{J}$, Kehlet $\mathrm{H}$. Secondary hyperalgesia to heat stimuli after burn injury in man. Pain. 1998;76(3):377-84.

29. Pereira MP, Werner MU, Ringsted TK, Rowbotham MC, Taylor BK, Dahl JB. Does naloxone reinstate secondary hyperalgesia in humans after resolution of a burn injury? A placebo-controlled, double-blind, randomized, cross-over study. PLoS One. 2013;8(5):e64608. doi:10. 61371/journal.pone.0064608. Print 0062013

30. Ravn P, Frederiksen R, Skovsen AP, Christrup LL, Werner MU. Prediction of pain sensitivity in healthy volunteers. J Pain Res. 2012;5:313-26.

31. Hansen MS, Wetterslev J, Pipper CB, Ostervig R, Asghar MS, Dahl JB. The Area of Secondary Hyperalgesia following Heat Stimulation in Healthy Male Volunteers. Inter- and Intra-Individual Variance and Reproducibility. PLoS One 2016, 11(5):e0155284.
32. McCormack K, Prather $P$, Chapleo C. Some new insights into the effects of opioids in phasic and tonic nociceptive tests. Pain. 1998;78(2):79-98.

33. Khambam SK, Naidu MU, Rani PU, Rao TR. A simple contact heat experimental pain model for evaluation of analgesic agents in healthy volunteers. Curr Ther Res Clin Exp. 2011;72(6):233-42.

34. Gottrup H, Andersen J, Arendt-Nielsen L, Jensen TS. Psychophysical examination in patients with post-mastectomy pain. Pain. 2000;87(3):275-84

35. Wright A, Moss P, Sloan K, Beaver RJ, Pedersen JB, Vehof G, et al. Abnormal quantitative sensory testing is associated with persistent pain one year after TKA. Clin Orthop Relat Res. 2015;473(1):246-54.

36. Hothorn T, Bretz F, Westfall P. Simultaneous inference in general parametric models. Biom J. 2008;50(3):346-63.

37. R Core Team (2014). R: A language and environment for statistical computing. R Foundation for Statistical Computing V, Austria. URL http:// www.R-project.org/.

38. Alabas OA, Tashani OA, Tabasam G, Johnson Ml. Gender role affects experimental pain responses: a systematic review with meta-analysis. Eur J Pain. 2012;16(9):1211-23.

39. Bartley EJ, Fillingim RB. Sex differences in pain: a brief review of clinical and experimental findings. Br J Anaesth. 2013;111(1):52-8.

40. Breimhorst M, Hondrich M, Rebhorn C, May A, Birklein F. Sensory and sympathetic correlates of heat pain sensitization and habituation in men and women. Eur J Pain. 2012;16(9):1281-92.

41. Campesi I, Fois M, Franconi F. Sex and gender aspects in anesthetics and pain medication. Handb Exp Pharmacol. 2012;214:265-78.

42. Choi JC, Chung Ml, Lee YD. Modulation of pain sensation by stress-related testosterone and cortisol. Anaesthesia. 2012;67(10):1146-51.

43. lacovides S, Avidon I, Baker FC. Does pain vary across the menstrual cycle? A review. Eur J Pain. 2015;19(10):1389-405. doi:10.1002/ejp.1714. Epub 2015 Apr 1321.

44. Mogil JS. Sex differences in pain and pain inhibition: multiple explanations of a controversial phenomenon. Nat Rev Neurosci. 2012;13(12):859-66.

45. Okifuji A, Hare BD. The association between chronic pain and obesity. J Pain Res. 2015:8:399-408.

46. Campbell CM, Edwards RR, Fillingim RB. Ethnic differences in responses to multiple experimental pain stimuli. Pain. 2005;113(1-2):20-6.

47. Edwards CL, Fillingim RB, Keefe F. Race, ethnicity and pain. Pain. 2001:94(2):133-7.

48. Edwards RR, Doleys DM, Fillingim RB, Lowery D. Ethnic differences in pain tolerance: clinical implications in a chronic pain population. Psychosom Med. 2001;63(2):316-23.

49. Rahim-Williams B, Riley 3rd JL, Williams AK, Fillingim RB. A quantitative review of ethnic group differences in experimental pain response: do biology, psychology, and culture matter? Pain Med. 2012;13(4):522-40.

50. Rahim-Williams FB, Riley 3rd JL, Herrera D, Campbell CM, Hastie BA, Fillingim RB. Ethnic identity predicts experimental pain sensitivity in African Americans and Hispanics. Pain. 2007:129(1-2):177-84.

51. Hinrichs-Rocker A, Schulz K, Jarvinen I, Lefering R, Simanski C, Neugebauer EA. Psychosocial predictors and correlates for chronic post-surgical pain (CPSP) - a systematic review. Eur J Pain. 2009;13(7):719-30.

52. Pincus T, Burton AK, Vogel S, Field AP. A systematic review of psychological factors as predictors of chronicity/disability in prospective cohorts of low back pain. Spine (Phila Pa 1976). 2002;27(5):E109-120.

53. Granot M, Ferber SG. The roles of pain catastrophizing and anxiety in the prediction of postoperative pain intensity: a prospective study. Clin J Pain 2005;21(5):439-45

54. Papaioannou M, Skapinakis P, Damigos D, Mavreas V, Broumas G, Palgimesi A. The role of catastrophizing in the prediction of postoperative pain. Pain Med. 2009;10(8):1452-9. doi:10.1111/j.1526-4637.2009.00730.x. Epub 02009 Oct 00726.

55. Hansen MS, Horjales-Araujo E, Dahl JB. Associations between psychological variables and pain in experimental pain models. A systematic review. Acta Anaesthesiol Scand. 2015;59(9):1094-102.

56. Salomons TV, Moayedi M, Erpelding N, Davis KD. A brief cognitivebehavioural intervention for pain reduces secondary hyperalgesia. Pain. 2014:155(8):1446-52.

57. Dyck PJ. Quantitative sensory testing: a consensus report from the Peripheral Neuropathy Association. Neurology. 1993;43(5):1050-2. 